\title{
Commentary and Reply
}

\section{Hemispheric symmetries in the identification of band-pass filtered letters: Reply to Christman et al. (1997)}

\author{
DAVID H. PETERZELL \\ University of Califormia, San Diego \\ La Jolla, Califormia
}

Christman, Kitterle, and Niebauer (1997) have examined the hypothesis that the two cerebral hemispheres are specialized for processing different ranges of spatial frequency. Their two experiments partially replicated an experiment of Peterzell, Harvey, and Hardyck (1989), who used Sergent's (1982) letter identification paradigm with spatial-frequency band-pass filtered letters as stimuli. We acknowledge the unusual strengths of Christman et al.'s experiments, but argue that the results support the original conclusion of Peterzell et al.: The results are not attributable to hemispheric asymmetries in spatial frequency processing.

\section{Unusual Methodological Strengths}

Christman, Kitterle, and Niebauer (1997) provide unusually important data regarding the relationship between visual spatial frequencies and hemispheric asymmetries. The crucial strength of their work lies in the fact that they (1) used band-pass filtered letters, (2) measured response bias and discriminability separately, and (3) differentiated proximal and distal spatial frequency.

Band-pass filtered patterns. Christman et al. (1997) note that many investigators have taken methodological shortcuts (e.g., stimulus blurring) to examine indirectly the relationship between spatial frequencies and the hemispheres.

Recognizing the limitations of these methodological shortcuts, Christman, Kitterle, and colleagues initially turned to using sine-wave grating stimuli (Christman, Kitterle, \& Hellige, 1991; Kitterle, Christman, \& Conesa, 1993; Kitterle, Christman, \& Hellige, 1990). By using gratings, they provided strong, direct evidence for a relationship between spatial frequency and hemispheric competence. They have demonstrated that, for certain tasks, the left visual field/right hemisphere (LVF/RH) processes $l o w^{\prime}$ spatial frequency gratings more rapidly and accurately than the right visual field/left hemisphere $(\mathrm{RVF} / \mathrm{LH})$, and that the RVF/LH processes high

Correspondence should be addressed to D. H. Peterzell. Department of Psychology. 0109. University of California, San Diego, La Jolla, CA 92093 (e-mail: peterzel(a)psy.ucsd.edu). spatial frequency gratings more rapidly and accurately than the LVF/RH.

The question remains whether the hemispheric asymmetries underlying grating processing can account for the many previously reported asymmetries. Because in previous experiments complex (nongrating) stimuli were used in conjunction with indirect manipulations of spatial frequency, the answer to this question is not obvious. At present, however, hemispheric asymmetries in spatial frequency processing are assumed to play a role in causing these previously reported asymmetries (Christman, 1989; Hellige, 1993; Sergent, 1987).

The rare experiments in which band-pass filtered stimuli have been used provide especially important tests of the spatial frequency hypothesis. Results of these experiments generalize readily to previous reports, unlike results from grating experiments (Christman et al., 1997; Peterzell, Harvey, \& Hardyck, 1989; Whitman \& Keegan, 1991). In these experiments, researchers have succeeded both in controlling spatial frequency directly (as do the grating experiments) and in using the complex stimuli (letters or faces) used previously. Because the filter bandwidths in these experiments are narrow ( 1 octave), there is explicit control over the spatial frequencies available in the input and explicit knowledge of the spatial frequencies required for performing an experimental task.

In particular, Peterzell et al. (1989) and Christman et al. (1997) used stimuli (letters) and procedures that were identical in most respects to those used by Sergent (1982; this was the original paper to suggest a link between spatial frequencies and the hemispheres). Thus, we can generalize our conclusions to Sergent's original, influential letter-identification study.

Response bias $(\beta)$ and discriminability $\left(d^{\prime}\right)$. Christman et al. (1997) are among the few to have measured response bias and discriminability separately in studies of hemispheric asymmetry (Chiarello, Nuding, \& Pollock, 1988; Chiarello, Senehi, \& Soulier, 1986; Kitterle et al., 1993; Peterzell et al., 1989). Over the years, many investigators have concluded that hemispheric specialization underlies asymmetries in performance. However, it is now apparent that response bias can account for some or all of the results, thus calling into question the interpretations of hundreds of studies. The hemispheres of the normal brain may be equally competent yet differ in their response criteria (Kitterle et al., 1993; Peterzell, 1991).

Proximal and distal spatial frequency. In only two previous studies have researchers examined whether proximal spatial frequency (cycles per degree of visual angle) or distal spatial frequency (cycles per object) is re- 
lated to hemispheric asymmetries, with conflicting results (Christman et al., 1991; Peterzell et al., 1989). The concept of distal spatial frequency was detailed by Peterzell et al. (1989, Figures 4 and 10).

In the experiment of Christman et al. (1997), distal spatial frequency may be reexpressed in cycles per letter. Stimuli contained 2 cycles/letter (large letters, 1-cpd filter; and small letters, 4 -cpd filter), 4 cycles/letter (large letters, 2-cpd filter; and small letters, 8-cpd filter), or 8 cycles/letter (large letters, 4-cpd filter; and small letters, 16-cpd filter). The reexpression of spatial frequency allows for proximal and distal spatial frequency hypotheses to be examined independently.

\section{Weak Empirical Support for Theory}

We suggest that the original conclusions of Peterzell et al. (1989) hold true for Christman et al.'s (1997) experiments, with important implications for the hemispheric spatial frequency hypothesis. Specifically, Christman et al.'s results do not support the spatial frequency hypothesis because: (1) the critical statistical interactions are weak, (2) spatial frequency is probably not the variable responsible for asymmetries, and (3) the asymmetries may reflect response criteria rather than competence.

Weak statistical interactions. Christman et al. (1997) note that "if the probability of making a Type I error had been set at .01 , as was done in the study by Peterzell et al., the only significant visual field effect in our data would have been the visual field $x$ spatial frequency $x$ target interaction in RT for the large-letter set in Experiment 2" (p. 283).

In fact, the conservative criterion of $\alpha=.01$ (chosen by Peterzell et al., 1989) seems especially appropriate, given the need to minimize experimentwise error. In order to hold the experimentwise error to .05 , the Bonferroniadjusted $\alpha$ criterion for each univariate test must be .003 for each of the four-way ANOVAs (assuming that one computes all main effects and interactions). Because only some statistcal comparisons are relevant to the spatial frequency hypothesis, a more lenient criterion of $\alpha=.01$ seems acceptable. However, the criterion of $\alpha=.05$ used by Christman et al. (1997) for univariate tests almost certainly led to Type I errors.

In other words, the differences between results of our study and Christman et al.'s (1997) seem to be due to nothing more than differing statistical decision criteria. It may be argued that the study of Christman et al., like that of Peterzell et al. (1989), contains little if any evidence for spatial frequency $\times$ visual field/hemisphere interactions.

Spatial frequency is probably not the critical variable. If one accepts the statistical criterion of $\alpha=.05$ used by Christman et al. (1997) despite warnings, one is left with a series of unsystematic, confusing statistical interactions. As Christman et al. acknowledge, these interactions fail to support the original hypothesis of Sergent (1982, 1987); the LVF/RH should have processed low spatial frequency letters more rapidly and accurately than the RVF/LH, and the RVF/L.H should have processed high spatial frequency letters more rapidly and accu- rately than the LVF/RH. Hence, Christman et al. conclude that the original spatial frequency hypothesis may be rejected.

While it is evident that a hypothesis based on proximal spatial frequency (cycles/degree) cannot explain the statistical interactions, it is also clear that a hypothesis based on distal spatial frequency (cycles/object) is equally unsuccessful. The distal or "relative" spatial frequency hypothesis makes predictions similar to Sergent's (1982, 1987) original hypothesis, except that spatial frequency is defined in terms of cycles per letter rather than cycles per degree, as described above (Christman et al., 1991; Peterzell et al., 1989). Had Christman et al. (1997) replotted and examined the data using cycles per letter rather than cycles per degree, the ANOVA results would not have changed. Moreover, the spatial frequency $\times$ visual field/hemisphere interactions would remain unsystematic and confusing. Hence, it seems fair to reject the distal spatial frequency hypothesis as well as Sergent's original hypothesis.

What, other than chance, might have caused the small, unsystematic, confusing interactions reported by Christman et al. (1997)? Insight may come by reexamining the study of Peterzell et al. (1989). In contrast to Christman et al., Peterzell et al. included in their stimulus set unfiltered stimuli as controls. These stimuli were normal, clearly focused letters of various sizes. No hemispheric differences were found for these unfiltered letters. In contrast, all band-pass filtered stimuli yielded higher hit rates in the right hemisphere than in the left, regardless of filter spatial frequency. The result clearly fails to support the hypothesis that hemispheric asymmetries are related to the specific spatial frequencies available and required for completing the task. Rather, the result implies that any interference with a letter's visibility can lead to hemispheric asymmetries. An explanation based on stimulus visibility per se is consistent with existing theory (Christman, 1989; Michimata \& Hellige, 1987; Peterzell, 1991; Sergent \& Hellige, 1986).

One may now return to previous reports, in which spatial frequency was manipulated indirectly (for a review, see Christman, 1989). In particular, it is worth reconsidering Sergent's (1982) original letter identification experiment. The widely held belief that asymmetries were due to spatial frequency seems unwarranted in light of the uncompelling results of Christman et al. (1997) and Peterzell et al. (1989). A variable other than spatial frequency, such as the general level of stimulus visibility, seems to be what caused performance asymmetries.

Hemispheric criterion, not hemispheric competence. Christman et al. (1997), unlike Peterzell et al. (1989), do find a systematic visual field/hemisphere $\times$ spatial frequency interaction for reaction times. The upper left panel of Figure 1 in Christman et al. shows the interaction predicted by the spatial frequency hypothesis; large letters containing low spatial frequencies are identified more rapidly in the LVF/RH than in the RVF/LH, whereas large letters containing high spatial frequencies are identified more rapidly in the RVF/LH than in the LVF/RH. 
Given these intriguing findings, Christman et al. (1997) write,

A number of factors suggest that it is unlikely that response biases could solely explain previous reports of visual field $X$ spatial frequency interactions. First, visual field $X$ spatial frequency interactions for RT data obtained in the present study held for both letter sets, even though the visual field $\times$ spatial frequency interaction for the $\beta$ measure was obtained for the small-letter set only. Second, as Christman (1993) pointed out, most previous reports of hemispheric differences in spatial frequency processing have been based on RT, a dependent measure less susceptible to response biases. (p. 283)

In fact, the results of Christman et al. (1997) are readily interpretable in terms of response biases. First, it should be noted that the claim that RT is "less susceptible to response biases" is incorrect. Response bias is a powerful determinant of response times, and it is not easily separated from other factors (Link, 1992; Luce, 1986).

Second, the dissimilarities between results for RT and $\beta$ can be reconciled with an explanation based on response biases. The results for RT and $\beta$ are quite possibly due to different varieties of response bias. The results for $\beta$ indicate that the LVF/RH was more disposed than the RVF/LH to respond that the letter presented was a target-a perceptual bias to see a certain class of stimuli. The results for RT certainly may have been influenced by this perceptual bias in complex, unknown ways. Equally likely, however, is that the RT results were influenced by biases related to the speed-accuracy tradeoff (Link, 1992; Luce, 1986). That is, a subject (or a cerebral hemisphere), instructed to respond as rapidly and accurately as possible, could choose to respond faster but with greater inaccuracy, or slower with greater accuracy. In short, the RT results may be due to asymmetries in criteria regarding the speed-accuracy trade-off, and not to the perceptual biases that influenced $\beta$.

As such, the discrepancies between the results for RTs and $\beta$ s are not informative. Response biases can provide the sole explanation for previous reports of visual field interactions.

\section{Concluding Remarks}

Many of the ideas discussed here are consistent with those expressed in the report of Christman et al. (1997). However, these ideas contradict the conclusion of Christman et al. That is, the two new experiments, like those of Peterzell et al. (1989), do not confirm the presence of hemispheric differences in spatial frequency processing.

\section{REFERENCES}

Chiarello, C., Nuding, S., \& Pollock, A. (1988). Lexical decision and naming asymmetries: Influence of response selection and response bias. Brain \& Language, 34, 302-314.

Chiarello, C., Senehi, J., \& Soulier, M. (1986). Viewing conditions and hemisphere asymmetry for the lexical decision. Neuropsychologia, 24, 521-529.

Christman, S. [D.] (1989). Perceptual characteristics in visual laterality research. Brain \& Cognition, 11, 238-257.

Christman, S. [D.], KitTerle, F. L., \& Hellige, J. [B.] (1991). Hemispheric asymmetry in the processing of absolute versus relative spatial frequency. Brain \& Cognition, 16, 62-73.

Christman, S. D., Kitterle, F. L. \& Niebauer, C. L. (1997). Hemispheric asymmetries in the identification of band-pass filtered letters. Psychonomic Bulletin \& Review, 4, 277-284.

Hellige, J. B. (1993). Hemispheric asymmetry: What's right and what's left. Cambridge, MA: Harvard University Press.

Kitterle, F. L., Christman, S. [D.], \& Conesa, J. (1993). Hemispheric differences in the interference among components of compound gratings. Perception \& Psychophysics, 54, 785-793.

Kitterle, F. L., Christman, S. [D.], \& Hellige, J. B. (1990). Hemispheric differences are found in the identification, but not the detection, of low versus high spatial frequencies. Perception \& Psychophysics, 48, 297-306.

LINK, S. W. (1992). The wave theory of difference and similarity. Hillsdale, NJ: Erlbaum.

LuCE, R. D. (1986). Response times: Their role in inferring elementary mental organization. Oxford: Oxford University Press.

Michimata, C., \& Hellige, J. B. (1987). Effects of blurring and stimulus size on the lateralized processing of nonverbal stimuli. Neuropsychologia, 25, 397-407.

PETERZELL, D. H. (1991). On the nonrelation between spatial frequency and cerebral hemispheric competence. Brain \& Cognition. 15, 62-68.

Peterzell, D. H., Harvey, L. O., JR., \& Hardyck, C. D. (1989). Spatial frequencies and the cerebral hemispheres: Contrast sensitivity, visible persistence, and letter classification. Perception \& PSychophysics, 46, 433-455.

SERGENT, J. (1982). The cerebral balance of power: Confrontation or cooperation? Journal of Experimental Psychology: Human Perception \& Performance, 8, 253-272.

SERGENT, J. (1987). Failures to confirm the spatial-frequency hypothesis: Fatal blow or healthy complication? Canadian Journal of Psychology, 41, 412-428.

Sergent, J., \& Hellige, J. B. (1986). Role of input factors in visualfield asymmetries. Brain \& Cognition, 5, 174-199.

Whitman, R. D., \& KeEGan, J. F. (1991). Lateralization of facial processing: A spatial frequency model. International Journal of Neuroscience, 60, 177-185.

(Manuscript received August 15, 1996; accepted for publication November 1, 1996.) 